Pneumologe 2013 · 10:311-311

DOI 10.1007/s10405-013-0671-x

Online publiziert: 25. August 2013

๑) Springer-Verlag Berlin Heidelberg 2013

Joachim Lorenz ${ }^{1} \cdot$ Bernhard Schaaf $^{2}$

${ }^{2}$ Klinikum Dortmund

${ }^{1}$ Klinik für Pneumologie und Internistische Intensivmedizin

(Infektiologie, Schlafmedizin), Klinikum Lüdenscheid

\title{
Respiratorische Virusinfektionen
}

Das Spektrum ambulant erworbener respiratorischer Infektionen reicht von der banalen Tracheobronchitis bis zur schweren Sepsis mit respiratorischem Versagen oder mit Multiorganversagen. Unter den nosokomialen Infektionen und den infektiösen Komplikationen bei Immuninkompetenz stellen Pneumonien die wichtigsten Manifestationen im Hinblick auf die Letalität dar.

Außer bei der nosokomialen Pneumonie sind virale Erreger bei allen Formen - vom "grippalen Infekt“ bis hin zu infektiösen Komplikationen in der Transplantationsmedizin - von erheblicher Bedeutung. Die vergangene Winterzeit hat mit einer schwerwiegenden Exzessletalität durch Influenza-A-Infektionen diesen Umstand erneut unterstrichen. Trotzdem gibt es eine Tendenz zum Neglect viraler Atemwegsinfektionen, wahrscheinlich als Folge beschränkter Optionen in der antiviralen Therapie. Gerade aber bei den schweren Formen der Influenza und bei den viralen opportunistischen Infektionen ist diese Skepsis nicht immer gerechtfertigt.

\section{》) Schwere virale Atemwegsinfektionen dürfen nicht vernachlässigt werden}

In den drei Beiträgen dieses Hefts werden wichtige Themen aus dem Bereich der respiratorischen Viruserkrankungen vorgestellt. Die Influenza gehört in vielen Jahren zu den häufigsten Ursachen einer ambulant erworbenen Pneumonie. In dem Beitrag von Marcus Panning werden eingehend die spezifischen Eigenschaften des höchst wandelbaren Virus dargestellt und eine kritische Bilanz über die therapeutischen Optionen gezogen. Die neuen und seltenen pneumotropen Viren sind ein spannendes Feld, das immer wieder hochaktuell wird. Auch der aktuellste Vertreter, das „Middle East Respiratory Syndrome Corona Virus", das uns im Rahmen der pneumologischen Reisemedizin jederzeit begegnen kann und das Ähnlichkeiten zum Erreger von SARS aus dem Jahre 2003 aufweist, wird vorgestellt. $D a$ niel Drömann gibt einen Überblick über Spektrum, Diagnostik und Therapie viraler Erkrankung der Atmungsorgane bei Immuninkompetenz.

Wir sind den Autoren für Beiträge dankbar, die eine Lücke in der pneumologischen Infektiologie schließen.

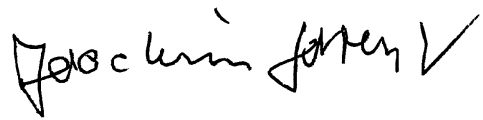

Joachim Lorenz

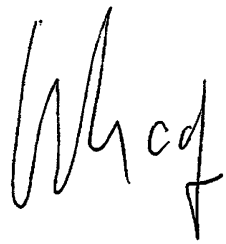

Bernhard Schaaf

\section{Korrespondenzadresse}

\section{Prof. Dr. J. Lorenz}

Klinik für Pneumologie und Internistische Intensivmedizin (Infektiologie, Schlafmedizin), Klinikum Lüdenscheid

Paulmannshöher Str. 14, 58515 Lüdenscheid joachim.lorenz@klinikum-luedenscheid.de 


\section{Galenus-von-Pergamon-Preis 2013}

\section{Herausragende Arzneimittel-Innovationen im Wettstreit um die begehrte Auszeichnung}

\section{Eklira $^{\circledast} /$ Bretaris $^{\circledast}$ \\ (Aclidiniumbromid)}

Aclidiniumbromid (Eklira ${ }^{\circledR}$ Genuair $^{\oplus}$ von Almirall; Bretaris ${ }^{\circledast}$ Genuair $^{\oplus}$ von Berlin-Chemie) ist ein langwirksamer Muskarin-Rezeptor-Antagonist mit raschem Wirkungseintritt. In klinischen Studien sind etwa 30 Minuten nach der ersten Inhalation Verbesserungen der Einsekundenkapazität ( $\mathrm{FEV}_{1}$ ) um 124 bis $133 \mathrm{ml}$ im Vergleich zum Ausgangswert messbar. Innerhalb von ein bis drei Stunden wird das Wirkmaximum mit FEV ${ }_{1}$-Steigerungen von bis zu $268 \mathrm{ml} \mathrm{im}$ Steady-State-Stadium erreicht. Weil Aclidiniumbromid im Plasma schnell abgebaut wird, ist die Rate systemischer anticholinerger Wirkungen gering.

Erwachsene mit COPD nehmen zur Linderung ihrer Symptome zweimal täglich $375 \mu \mathrm{g}$ Aclidiniumbromid (entspricht $322 \mu \mathrm{g}$ Aclidinium) mit Hilfe des Multidosis-Pulverinhalators (Genuair ${ }^{\oplus}$ ) ein. In Studien verringerte die Substanz frühmorgendliche Symptome wie Kurzatmigkeit nach dem Aufstehen sowie nächtliche Symptome. Auch moderate oder schwere COPD Exazerbationen traten deutlich seltener auf.

\section{Esbriet $^{\circledR}$}

\section{(Pirfenidon)}

Für Patienten mit leichter bis mittelschwerer idiopathischer Lungenfibrose (IPF) ist mit Esbriet ${ }^{\oplus}$ (Pirfenidon) von InterMune erstmals ein spezifisch wirksames Medikament erhältlich. Das Arzneimittel ist seit 2011 EU-weit zugelassen.

Pirfenidon hat antifibrotische, entzündungshemmende und antioxidative Eigenschaften. Die Substanz verzögert die weitere, den Gasaustausch behindernde
Fibrosierung. Dadurch wird die progrediente Abnahme der Lungenfunktion verlangsamt. Im CAPACITY-Studienprogramm bremste Pirfenidon den Verlust der forcierten Vitalkapazität. Die Abnahme der Belastungstoleranz konnte aufgehalten werden. Außerdem gelang es, die progressionsfreie Überlebenszeit zu verlängern.

Experten haben in der im Februar 2013 veröffentlichten S2K-Leitlinie zur Diagnostik und Therapie bei IPF der Substanz als einzigem Arzneimittel eine schwach positive Empfehlung ausgesprochen, dagegen wurden andere medikamentöse Therapiestrategien aufgrund neuerer Erkenntnisse durchweg negativ bewertet.

\section{Kalydeco $^{\mathrm{TM}}$ \\ (Ivacaftor)}

Mit Ivacaftor (Kalydeco ${ }^{\mathrm{TM}}$ ) von Vertex Pharmaceuticals ist im Juli 2012 erstmals ein kausal wirkendes Medikament für Patienten mit zystischer Fibrose (CF; Mukoviszidose) zugelassen worden, und zwar für Patienten ab sechs Jahren mit einer G551D-Mutation im CFTR-Gen (cystic fibrosis transmembrane conductance regulator). Ivacaftor erhöht die Öffnungswahrscheinlichkeit des G551D-CFTR-Kanals und verstärkt so den Chlorid-Ionen-Transport. Die sich daraus ergebenden Veränderungen der Drüsensekrete verbessern die mukoziliäre Clearance an der Epitheloberfläche.

Unter oraler Therapie mit dem neuen Wirkstoff verbesserten sich in Studien innerhalb von zwei Wochen nachhaltig die Lungenfunktion und das Atemvolumen. Zudem nahmen die Patienten an Körpergewicht zu, und auch die Lebensqualität stieg.
Aufgrund der positiven Studienergebnisse misst der Gemeinsame Bundesausschuss (GBA) der Behandlung mit Ivacaftor besonders für Jugendliche und Erwachsene mit Mukoviszidose einen beträchtlichen Zusatznutzen bei.

\section{Sinupret ${ }^{\circledR}$ extract $^{2}$}

Sinupret ${ }^{\oplus}$ extract - ein hoch konzentrierter Trockenextrakt aus Enzianwurzel, Schlüsselblumenblüten, Ampferkraut, Holunderblüten und Eisenkraut von Bionorica SE - beschleunigt die Genesung von Patienten mit akuter unkomplizierter Rhinosinusitis. In Studien konnten nicht nur sekretolytische und antiinflammatorische Effekte, sondern auch antivirale Wirkungen gegen Erkältungsviren und eine signifikant verkürzte Genesungszeit nachgewiesen werden.

Der konzentrierte Extrakt ist eine Weiterentwicklung des pflanzlichen Kombinationspräparates Sinupret ${ }^{\oplus}$. Statt bislang $156 \mathrm{mg}$ Pflanzenmischung in Sinupret ${ }^{\circledR}$ forte wird nun eine vierfach höher dosierte Ausgangsdroge (720 mg) verwendet, aus der $160 \mathrm{mg}$ Trockenextrakt entstehen. Daraus resultiert eine im Vergleich 3,3-fach höhere Konzentration an Bioflavonoiden. Hintergrund ist die in Studien festgestellte Dosisabhängigkeit der pharmakologischen Wirkungen. Das Präparat ist zugelassen für Erwachsene mit akuten, unkomplizierten Entzündungen der Nasennebenhöhlen.

Quelle und weitere Infos: www.aerztezeitung.de 
Hier steht eine Anzeige.

算 Springer 